\title{
Perfil sociodemográfico e clínico de uma equipe de enfermagem portadora de Hipertensão Arterial
}

Sociodemographic and clinical profile of a nursing team with high blood pressure

Perfíl sociodemografico y clínico de un eQuipo de enfermería con presión alta

\author{
Ires Lopes Custódio', Francisca Elisângela Teixeira Lima", \\ Maria Irismar de Almeida'II, Lúcia de Fátima da Silva', Ana Ruth Macedo Monteiro' \\ 'Universidade Estadual do Ceará. Hospital de Mesejana. Fortaleza, CE \\ "Universidade Federal do Ceará. Departamento de Enfermagem da Faculdade de Farmácia, Odontologia e Enfermagem. Fortaleza, CE \\ ill Universidade Estadual do Ceará, Fortaleza, CE
}

Submissão: 14/08/2009

Aprovação: 20/11/2010

\section{RESUMO}

Estudo descritivo-exploratório desenvolvido em um hospital terciário de Fortaleza, CE, Brasil com objetivo de avaliar as características dos profissionais de enfermagem com hipertensão arterial em um hospital de doenças cardiovasculares. Em população constituída pela equipe de enfermagem, a amostra continha 130 participantes. Os dados foram coletados por meio de entrevista e as características sociodemográficas predominantes foram: nível médio (72,3\%); sexo feminino (79,2\%); idade > 40 anos (79,2\%); casados (41,5\%) e não-brancos $(65,4 \%)$. Fatores de risco não modificáveis: história familiar $(71,5 \%)$. Os modificáveis foram: diabetes mellitus $(24,6 \%)$, tabagismo (35,4\%); etilismo (43,8\%); dieta inadequada (68,5\%); dislipidemia (24,6\%) e sedentarismo $(62,3 \%)$. É necessário desenvolver estratégias de educação em saúde e programar acompanhamento desses profissionais de saúde, visando controlar a pressão arterial e evitar complicações cardiovasculares.

Descritores: Hipertensão; Perfil de saúde; Fatores de risco; Enfermagem do trabalho.

\section{ABSTRACT}

Descriptive-exploratory study carried out in a hospital from Fortaleza, CE, Brazil to evaluate the characteristics of nursing professionals with High Blood Pression from a hospital for cardiovascular diseases. In the population of the nursing staff, the sample had 130 participants, who met the criteria for inclusion. Data were collected through interviews and sociodemographic characteristics were predominant: average level (72.3\%), female (79.2\%), age > 40 years (79.2\%), married (41.5\%) and non-whites (65.4\%). Non-modifiable risk factors identified: familial history (71.5\%). The modifiable were: diabetes mellitus (24.6\%), smoking (35.4\%), alcoholism (43.8\%), inadequate diet (68.5\%), hyperlipidemia (24.6\%) and sedentary (62.3\%). It is be necessary to develop strategies for health education and program monitoring of health professionals, in order to control blood pressure and preventing cardiovascular complications.

Key words: Hypertension; Health profile; Risk factors; Occupational health nursing.

\section{RESUMEN}

Estudio descriptivo-exploratorio desarrollado en un hospital terciario de Fortaleza, CE, Brasil para evaluar las características de los profesionales de enfermería con la hipertensión arterial en un hospital para enfermedades cardiovasculares. En la población del personal de enfermería, la muestra de 130 participantes, Que cumplieron los criterios de inclusión. Los datos fueron recolectados a través de entrevistas y características sociodemográficas predominaron: nivel medio $(72,3 \%)$, mujeres $(79,2 \%)$, edad $>40$ años $(79,2 \%)$, casadas $(41,5 \%)$ y no blancos $(65,4 \%)$. No identificado factores de riesgo modificables: antecedentes familiares $(71,5 \%)$. El modificables fueron: diabetes mellitus $(24,6 \%)$, tabaco $(35,4 \%)$, alcoholismo $(43,8 \%)$, dieta inadecuada $(68,5 \%)$, dislipidemia $(24,6 \%)$ y sedentarismo $(62,3 \%)$. Es necesario desarrollar estrategias de educación para la salud y programa de vigilancia de los profesionales de la salud, a fin de controlar la presión arterial y la prevención de complicaciones cardiovasculares.

Descriptores: Hipertensión arterial; Perfíl de salud; Factores de riesgo; Enfermería del trabajo. 


\section{INTRODUÇÃO}

Diferentemente do ocorrido por longo tempo, hoje inúmeras políticas públicas estão sendo elaboradas, voltadas, sobretudo, à saúde da população, principalmente com o intuito de melhorar a assistência às pessoas por meio de ações de promoção, proteção e recuperação da saúde. Urge, porém, desenvolver ações centradas na saúde do trabalhador, em virtude dos elevados índices de morbimortalidade presentes nos profissionais da saúde, em especial as doenças crônico-degenerativas, a exemplo das cardiovasculares.

Dentre as doenças cardiovasculares, destaca-se a Hipertensão Arterial Sistêmica (HAS), patologia capaz de afetar as estruturas físicas e psicossociais dos indivíduos. Isso é mais acentuado Quando se trata de profissionais da enfermagem que atuam na assistência aos pacientes portadores de doenças cardiovasculares, mostrando a necessidade da avaliação da hipertensão arterial desses profissionais, por conhecerem as formas de tratamento e complicações da hipertensão.

A HAS possui diversos fatores de risco, tanto modificáveis Quanto não modificáveis. Como não modificáveis mencionam-se os seguintes: história familiar de doença coronariana; idade avançada; sexo masculino e raça negra. Quanto aos modificáveis, incluem: dislipidemia; tabagismo; etilismo, nível sanguíneo de glicose elevada; obesidade; sedentarismo; estresse, má alimentação e uso de contraceptivo $^{(1)}$.

A literatura tem descrito a associação entre trabalho profissional, doenças cardiovasculares e a influência do trabalho na elevação da pressão arterial ${ }^{(2)}$. Nos dias atuais, as pessoas estão trabalhando com carga horária Que lhe tomam praticamente todo o dia, não restando condições para usufruírem de atividades prazerosas como o lazer, prática de exercícios, educação, cultura, boa alimentação e descanso, no Qual são acessórios importantes para Qualidade de vida e promoção de saúde.

Estudos com profissionais de enfermagem identificaram fatores favoráveis ao desencadeamento de doenças, como sobrecarga de trabalho, dificuldade em delimitar os diferentes papéis, problemas nas relações interpessoais, carga emocional, recursos inadequados e falta de poder de decisão e de reconhecimento ${ }^{(3)}$.

A enfermagem é reconhecida como uma atividade humanitária e de intenso trabalho, Que desempenha sua assistência voltada para o cuidar, sobretudo lida com situações de sofrimento, dor e morte em diversos momentos dos horários laborais.

Contudo, no processo do cuidar a equipe de enfermagem atua como cuidador do outro, e na maioria das vezes se esquece de praticar o autocuidado. Vários fatores concorrem para essa problemática, entre estes, a dupla jornada de trabalho, a responsabilidade, muitas vezes de garantir a manutenção da família, e outros compromissos referentes às condições econômicas. Todas contribuem para o surgimento da hipertensão arterial, incluindo, ainda, o estresse advindo do ambiente de trabalho e o constante desgaste emocional em face desse convívio.

Segundo evidenciado, alguns membros da equipe de enfermagem, já manifestaram complicações em decorrência da hipertensão arterial durante as atividades laborais, tais como: acidente vascular encefálico, angina instável e infarto agudo do miocárdio, bem como se depararam com crise de hipertensão moderada, por falta de acompanhamento e tratamento regular.
Diante dessas considerações, busca-se responder aos Questionamentos: Quais as características sociodemográficas dos profissionais da equipe de enfermagem portadores de hipertensão arterial? Que fatores de risco da hipertensão estão presentes? Os profissionais participam de programas educativos de hipertensão arterial?

Conforme se acredita, a resolução dessas Questões poderá sensibilizar os profissionais de enfermagem Quanto à importância de alguns aspectos, tais como: realização dos exames periódicos, tratamento regular da hipertensão e modificações no estilo de vida. Dessa forma, espera-se contribuir para adoção de um comportamento mais saudável, favorecendo o controle da HAS nesses profissionais, além de evitar mais casos de hipertensão arterial.

Tem-se, então, como objetivo geral: avaliar as características dos profissionais de enfermagem com HAS em um hospital de doenças cardiovasculares. E específicos: identificar o perfil sociodemográfico da equipe de enfermagem portadora de HAS; verificar os fatores de risco modificáveis e não-modificáveis para HAS presentes nos profissionais de enfermagem; averiguar a participação dos profissionais de enfermagem em programas educativos de HAS.

\section{MÉTODOS}

Tratou-se de um estudo descritivo-exploratório desenvolvido em uma instituição pública de saúde, um hospital de nível terciário, com referência em atendimento cardiovascular para as regiões do norte e nordeste do país, conveniado ao Sistema Único de Saúde, situado em Fortaleza, CE. O mencionado hospital dispõe de 306 leitos, dos Quais 39 pertencem à unidade de terapia intensiva para adultos, 12 pediátricas, 241 leitos clínicos e 14 semi-intensivos.

Da população do estudo fizeram parte 278 enfermeiros, 219 técnicos de enfermagem e $42 \mathrm{I}$ auxiliares de enfermagem Que trabalham na referida instituição. A amostra foi composta aleatoriamente e constituída por 130 membros da equipe de enfermagem, a saber: enfermeiros, técnicos e auxiliares de enferma-gem, Que atenderam aos critérios de inclusão: trabalhar na instituição, prestando assistência de enfermagem ao cliente; ser portador de hipertensão arterial; ter idade superior a 18 anos; estar trabalhando no período da coleta de dados; e apresentar condições físicas e emocionais para responder aos Questionamentos. Como critérios de exclusão, constaram: estar de férias ou licença no período de coleta de dados e não fazer parte do euadro assistencial.

A coleta de dados foi realizada em outubro de 2008, por meio de uma entrevista, com a utilização de um roteiro contendo perguntas abertas e fechadas, relacionadas às características sociodemográficas, ao conhecimento da doença e seus fatores de risco, as condutas terapêuticas, e à participação em programas educativos para hipertensão arterial.

De posse dos resultados, estes foram apresentados e analisados de forma estatística e descritiva, e tabulados em tabelas no programa Excel do Windows XP Professional.

Em atendimento ao exigido, a aceitação dos profissionais de enfermagem em participar do estudo foi registrada no termo de consentimento livre e esclarecido. O estudo seguiu os preceitos da Resolução no 196/96 do Conselho Nacional de Saúde ${ }^{(4)}$. Para tanto, o projeto foi aprovado pelo Comitê de Ética e Pesquisa do referido hospital, sob parecer $n^{\circ} 548 / 08$. 
Os dados obtidos foram analisados por meio de estatística descritiva.

\section{RESULTADOS}

Caracterização dos profissionais segundo a categoria profissional e características sociodemográficas

O grupo estudado, composto por 130 profissionais da equipe de enfermagem portadores de HAS, foi assim distribuído: 36 $(27,7 \%)$ enfermeiros, $38(29,2 \%)$ técnicos e $56(43,1 \%)$ auxiliares de enfermagem.

$\mathrm{Na}$ Tabela I constam os dados concernentes à atividade laboral na referida instituição dos 130 participantes do estudo. Em relação ao grau de escolaridade, $72,3 \%$ possui nível médio, representados pelos auxiliares e técnicos de enfermagem. Dos 36 enfermeiros, $6,2 \%$ possuíam somente graduação em enfermagem, $20 \%$ fez uma especialização, 1,5\% concluiu mestrado. Como observado, nenhum participante do estudo tinha doutorado.

Quanto ao tempo de trabalho na instituição, segundo identificado, variou de seis meses a 25 anos, com uma média de 17 anos. Constatou-se um predomínio de pessoas com hipertensão arterial nas unidades fechadas $(45,4 \%)$, seguidas pelo setor de emergência $(36,1 \%)$ e pelas unidades abertas, com 18,5\% desses profissionais. Foram consideradas unidades fechadas: unidades de terapia intensiva, semi-intensiva e centro cirúrgico; e unidades abertas: enfermarias clínicas e cirúrgicas. Estes aspectos são relevantes, pois os profissionais Que trabalham com pacientes críticos possuem maior carga de estresse, fator favorável para elevação da pressão arterial.

Ainda, como os achados da pesquisa mostraram, esses profissionais trabalham com escala de serviço em plantões alternados entre diurnos e noturnos (32,3\%), em somente diurnos (30,8\%), em plantões noturnos $(25,4 \%)$ e em escala diária nos dias úteis (1 1,5\%). Ressalta-se com esses dados Que menos de um Quarto prefere trabalhar somente no horário noturno, enQuanto a maioria prefere trabalhar no horário diurno.

Com base na Tabela 2, verifica-se dentre os 130 entrevistados a predominância do sexo feminino (79,2\%), e apenas $20,8 \%$ do sexo masculino. A idade dos participantes variou de 23 a 65 anos, com uma média de 45 anos de idade.

Quanto ao estado civil, segundo se observou no estudo, a maioria dos entrevistados $(41,5 \%)$ eram casados; 27 (20,8\%) solteiros, 36 $(27,7 \%)$ separados e $13(10 \%)$ viúvos. Em relação à cor da pele, $85(65,4 \%)$ referiram ter cor da pele não-branca e $45(34,6 \%)$ se consideram da cor branca.

Fatores de risco não-modificaveis e modificáveis presentes nos profissionais de enfermagem

Em relação à hereditariedade, fator de risco não-modificável para HAS, como informamos, $93(71,5 \%)$ profissionais tinham história familiar da doença; 20 (15,4\%) não tinham esse fator hereditário; e 17 (13, 1\%) não sabiam de casos na família com a HAS (Tabela 3).

Por ser uma doença crônica, a Diabetes Mellitus não tem cura, apenas controle. Portanto, é um fator de risco não-modificável. Embora esta patologia tenha sido identificada em 32 (24,6\%) profissionais, a maioria $(66,9 \%)$ afirmou não ser portador e 1 I $(8,5 \%)$ não sabe se é portador. No caso das doenças crônicas, existem dois fatores principais de complicações: o diagnóstico tardio e o déficit de conhecimento.

Em prosseguimento à análise dos dados do estudo, a prática do tabagismo foi detectada em 66 pessoas, as Quais confirmaram já terem sido fumantes em alguma época de suas vidas. Como evidenciado, os efeitos do tabagismo são maléficos em curto ou longo prazo para a saúde. Destas pessoas, $46(35,4 \%)$ permanecem fumando cigarros, $20(15,4 \%)$ pararam e as demais $(49,2 \%)$ relataram jamais terem fumado.

No referente ao etilismo, foram identificados 84 profissionais. Eneuanto $57(43,8 \%)$ referiram ainda serem consumidores de álcool etílico, embora apenas socialmente, ou seja, uma ou duas vezes por semana, uma garrafa de cerveja, 27 (20,8\%) informaram Que pararam de ingerir álcool. O consumo do álcool, de certa maneira, eleva a pressão arterial tanto aguda Quanto cronicamente.

Tabela 1. Caracterização dos profissionais segundo titulação, tempo de trabalho, setor, turno de trabalho. Fortaleza, CE, 2008.

\begin{tabular}{lcc} 
Características laborais & $\mathbf{n}$ & \% \\
\hline Titulação & 94 & 72,3 \\
Ensino médio & 08 & 6,2 \\
Graduação & 26 & 20 \\
Especialização & 02 & 1,5 \\
Mestrado & & \\
Tempo de Trabalho & 29 & 22,3 \\
$<1$ ano & 15 & 11,5 \\
||$-3$ anos & 18 & 13,8 \\
$3 \mid-5$ anos & 28 & 21,5 \\
$5 \mid-10$ anos & 40 & 30,9 \\
$>10$ anos & & \\
Setor & 59 & 45,4 \\
Unidade Fechada & 24 & 18,5 \\
Unidade Aberta & 47 & 36,1 \\
Emergência & & \\
Turno de trabalho & 42 & 32,3 \\
Plantão (diurno/noturno) & 40 & 30,8 \\
Plantão (diurno) & 33 & 25,4 \\
Plantão (noturno) & 15 & 11,5 \\
Diarista &
\end{tabular}

Tabela 2. Caracterização dos profissionais segundo sexo, faixa etária, estado civil e cor. Fortaleza, CE, 2008.

\begin{tabular}{lcc} 
Características demográficas & $\mathbf{n}$ & \% \\
\hline Sexo & 103 & 79,2 \\
Feminino & 27 & 20,8 \\
Masculino & & \\
Faixa Etária (anos) & 04 & 3,7 \\
$20-30$ & 23 & 17,7 \\
$30 \mid-40$ & 47 & 36,1 \\
$40 \mid-50$ & 42 & 32,3 \\
$50 \mid-60$ & 14 & 10,8 \\
$\geq 60$ & & \\
Estado Civil & 54 & 41,5 \\
Casado & 27 & 20,8 \\
Solteiro & 13 & 10,0 \\
Viúvo & 36 & 27,7 \\
Separado & & \\
Cor & 45 & 34,6 \\
Branca & 85 & 65,4 \\
Não-Branca & & \\
\hline
\end{tabular}


Tabela 3. Caracterização dos profissionais de enfermagem Quanto à presença de fatores de risco, considerando como indicadores: hereditariedade, Diabetes Mellitus, tabagismo, etilismo, dieta inadequada, dislipidemia e sedentarismo. Fortaleza, CE, 2008.

\begin{tabular}{lcc}
\hline Características Demográficas & $\mathbf{n}$ & \% \\
\hline Hereditariedade & 93 & 71,5 \\
Sim & 20 & 15,4 \\
Não & 17 & 13,1 \\
Não sabe & & \\
Diabetes Mellitus & 32 & 24,6 \\
Sim & 87 & 66,9 \\
Não & 11 & 8,5 \\
Não Sabe & & \\
Tabagismo & 46 & 35,4 \\
Sim & 20 & 15,4 \\
Parou & 64 & 49,2 \\
Nunca & & \\
Etilismo & 57 & 43,8 \\
Sim & 27 & 20,8 \\
Parou & 46 & 35,4 \\
Nunca & & \\
Dieta InadeQuada & 89 & 68,5 \\
Sim & 41 & 31,5 \\
Não & & \\
Dislipidemia & 32 & 24,6 \\
Sim & 35 & 42,3 \\
Não & 53 & 33,1 \\
Não sabe & 43 & \\
Sedentarismo & 49,3 \\
Sim & & 37,7 \\
Não & & \\
\hline
\end{tabular}

Tabela 4. Caracterização dos profissionais de enfermagem segundo a participação em programas educativos para hipertensão arterial e realização de exame periódico na instituição. Fortaleza, CE, 2008.

\begin{tabular}{lcc}
\hline Ações de Promoção da Saúde & $\mathbf{n}$ & \% \\
\hline Programa Educativo & 3 & 2,3 \\
Sim & 127 & 97,7 \\
Nunca & & \\
Exame Periódico & 38 & 29,3 \\
Sim & 64 & 49,2 \\
Não & 28 & 21,5 \\
Iniciativa Própria & & \\
\hline
\end{tabular}

Sobre a dieta, $41 \quad(31,5 \%)$ participantes aderem a uma dieta saudável, contudo, foram citadas várias dificuldades pelos profissionais para manterem uma alimentação saudável, entre estas, a carga horária de trabalho elevada, levando-os a alimentar-se em local não-domiciliar, o Que favorece a ingestão de produtos industrializados, ricos em gorduras e carboidratos e pobre em frutas, legumes e verduras. Dessa forma, torna-se impossível o controle dos tipos de alimentação, sobretudo realizar uma educação alimentar em seu cotidiano.

Ainda como identificado a dislipidemia estava presente em 32 (24,6\%) profissionais portadores de hipertensão arterial; 55 (42.3\%) negaram a existência deste fator de risco; e $43(33,1 \%)$ desco- nhecem a presença de dislipidemia.

Outro fator de risco analisado foi o sedentarismo. De acordo com a resposta dos entrevistados 81 (62,3\%) deles não praticavam exercícios. Destes, $20(24,7 \%)$ nunca praticaram, enQuanto 49 $(37,7 \%)$ praticavam regularmente exercícios, tais como: caminhada e atividade aeróbica em academia, de três a Quatro vezes por semana.

Caracterização dos profissionais segundo a participação em programas educativos e realização de exames

Como mostra a Tabela 4, 97,7\% dos profissionais entrevistados nunca participou de programas educativos para HAS e somente 2,3\% mencionou sua participação em programas educativos promovidos pela própria instituição ou em outros locais.

Esses programas educativos envolvem a participação das pessoas com HAS e contemplam ações de educação em saúde, direcionadas ao controle da pressão arterial a partir do conhecimento da doença e seus agravos de saúde, contemplando também assuntos como orientação nutricional, prática de exercícios físicos, controle do peso, e principalmente estimulando para o autocuidado e adesão ao estilo de vida saudável.

Quanto à realização dos exames periódicos na instituição, 64 $(49,2 \%)$ não realizam, apesar da existência do Serviço Especializado em Engenharia de Segurança e em Medicina do Trabalho (SESMT), Que é regido pela norma regulamentadora (NR) $n^{\circ} 4^{(5)}$.

Dos 66 profissionais Que relataram realizar exame periodicamente, $28(21,5 \%)$ o fazem por iniciativa própria e 38 $(29,3 \%)$ conseguiram na instituição, após a implantação do SESMT.

\section{DISCUSSÃO}

Os achados constataram a alta predominância (43, 1\%) de HAS nos auxiliares de enfermagem. Tais dados condizem com um estudo desenvolvido em Salvador, BA, Que constatou também uma predominância da hipertensão arterial nos auxiliares de enfermagem, perfazendo um percentual de $40,1 \%$, este fato pode ser decorrente do maior Quantitativo da equipe de enfermagem ser desse profissional.

Ressalta-se ainda, Que a dimensão da problemática acerca da hipertensão arterial e a magnitude envolvendo a amostra estudada no presente estudo, surpreendem pelos altos índices de hipertensão presentes nesses profissionais. Tal dado ocorre por se tratar de um grupo ocupacional da saúde, no Qual seria esperado encontrar menor prevalência da doença, em virtude da equipe de enfermagem ter oportunidade de adQuirir informações acerca da patologia, tratamento e prevenção, e ainda tem o papel de disseminá-las. Especialmente, por se tratar de profissionais Que trabalham em um hospital de referencia nas áreas cardiovascular, torácica e pulmonar, e atuar como centro de ensino e pesquisa.

O referido hospital promove assistência à população do Estado do Ceará, de forma humanizada e de Qualidade, contemplando programas de Qualidade de vida direcionados aos clientes e funcionários. Para tanto mantém uma equipe de saúde ocupacional para atender a todos os trabalhadores da instituição(7).

Nessa ótica, esses profissionais são agentes privilegiados, pois além de vivenciar a realidade de pacientes com complicações das doenças cardiovasculares, fazem parte de uma equipe Que articula 
os fatores imprescindíveis para a promoção da saúde.

Outro fator importante é a Questão do autocuidado. Em estudo realizado para investigação da prática do autocuidado vivenciada pela mulher hipertensa, evidenciou-se déficit de conhecimento e adesão parcial ao tratamento, bem como prática ineficaz do autocuidado, sobretudo era influenciada pela idade, pelo estágio de desenvolvimento e por problemas sociais, financeiros e de saúde ${ }^{(8)}$.

Nesse estudo, evidenciou-se que a maioria dos profissionais apresenta escalas mistas de trabalho, a saber: plantões de doze horas, no período diurno e noturno, com frequente alternância dos turnos. Em se tratando dos locais de trabalho da instituição, a maioria $(81,5 \%)$ atua em unidades de cuidados aos pacientes críticos, como unidades de terapia intensiva e emergências. Esse fato pode ser decorrente das melhorias salariais, referentes às políticas de gratificação para profissionais Que desempenham suas atividades em ambientes de cuidados intensivos. Além das condições econômicas mais favoráveis, há maior disponibilidade de material e equipamento para o desempenho dos serviços, bem como à segurança do profissional. Entretanto isso se faz contraditório à oratória da sobrecarga de trabalho e a falta de tempo para cuidar-se.

Nas análises de estudos sobre a relação do trabalho da enfermagem em unidades críticas e suas repercussões na saúde dos trabalhadores, a equipe de enfermagem integra uma das parcelas de trabalhadores de saúde Que estão, cotidianamente, expostos às cargas de trabalho excessivas, ou seja, exigências ou demandas psicobiológicas do processo de trabalho passiveis de gerar, ao longo do tempo, desgaste das capacidades vitais do trabalhador ${ }^{(9)}$. Tal afirmação corrobora com o nosso estudo Que constatou um predomínio de pessoas com hipertensão arterial nas unidades fechadas e setor de emergência.

Preocupa, ainda, a exposição dessa categoria aos riscos, derivados do ambiente de trabalho e das atividades laborais, pois estes interferem diretamente na saúde e devem ser permanentemente monitorados pela equipe de segurança e saúde ocupacional. As condições precárias de trabalho e o achatamento salarial, associados às más condições de vida, agravam ainda mais a situação, sujeitando os profissionais a manter mais de um vínculo de trabalho e a uma carga mensal longa e desgastante $\mathrm{e}^{(10)}$.

Ao analisar as relações de gênero, confirmou-se a participação dominante do sexo feminino $(79,2 \%)$ na equipe de enfermagem, evidenciando uma feminilização do trabalho dessa equipe, corroborando com um estudo realizado com a equipe de enfermagem sobre gênero e trabalho noturno, o Qual também constatou predomínio de mulheres Que trabalham a noite ${ }^{(1)}$.

Quanto à faixa etária, a prevalência da hipertensão arterial aumenta progressivamente com a idade, sendo superior a $50 \%$ entre os idosos. Contudo, esse estudo mostrou Que 79,2\% dos profissionais estão na faixa etária correspondente ao adulto (40 a 59 anos) e idoso ( $\geq 60$ anos). Tais dados podem ser decorrentes de ter sido realizado com profissionais economicamente ativos ${ }^{(12)}$.

E em relação à cor, a grande parte dos participantes desse estudo tem a cor da pele não-branca (pardas e negras), somando um percentual de $65,4 \%$. Tais dados condizem com um estudo desenvolvido em Salvador-BA, o Qual constatou Que as mulheres pardas ou negras apresentaram Quase duas vezes mais hipertensão arterial do Que as brancas, $38,7 \%$ e $22,1 \%$, respectivamente ${ }^{(6)}$.
Como fatores de risco não-modificáveis, além da idade, sexo e raça, citados anteriormente, tem-se a hereditariedade de HAS, cujos achados mostraram o predomínio de 71,5\% com história positiva de hipertensão na família, condizendo com o estudo desenvolvido no sul do Brasil, o Qual encontrou um índice de $56,3 \%$ de prevalência da hipertensão tanto paterna Quanto materna dos sujeitos estuda$\operatorname{dos}^{(13)}$.

Entre os fatores modificáveis, a dieta inadequada e o padrão sedentário de vida estavam presentes na maioria dos profissionais de enfermagem participantes do estudo.

Em relação à dieta, atualmente o mundo oferece dietas industrializadas consideradas favoráveis aos fatores de riscos para a hipertensão. Agravados pelo sedentarismo, esses fatores contribuem para a elevação de riscos para as complicações tardias e imediatas da doença ${ }^{(14)}$.

Outro aspecto relevante a ser considerado na dieta como fator de risco é o uso do sal, principalmente Quando adicionado à comida, no momento da refeição( ${ }^{(13)}$.

Conforme amplamente divulgado, a prática de atividade física é necessária e importante. Contudo, deve-se avaliar a frequência e a intensidade dos exercícios, pois nos estudos, dos autores ora citados, embora a maioria dos entrevistados $(71,9 \%)$ praticasse alguma atividade física com regularidade, apresentavam sobrepeso ${ }^{(14)}$.

Um estudo, ao buscar a prática de exercícios como um fator benéfico para o estilo de vida e a prevenção e/ou controle dos níveis pressóricos, detectou Que os homens praticavam esporte no final de semana jogando futebol de salão, já as mulheres não praticavam esportes e não exerciam QualQuer tipo de atividade Que prevenisse o excesso de peso e, conseqüentemente, a hipertensão arterial, confirmando a prevalência do sedentarismo na população urbana brasileira é maior em mulheres do Que em homens ${ }^{(15)}$.

Prevenir e tratar a hipertensão arterial envolve ensinamentos para o conhecimento da doença, de suas inter-relações, de suas complicações e implica, na maioria das vezes, a exigência de mudanças de hábitos de vida ${ }^{(16)}$.

Ao tratar a hipertensão deve-se ter em mente os fatores de risco associados e o impacto do tratamento nestes fatores. Assim, apesar de um controle satisfatório da hipertensão, outros fatores de risco potencialmente maiores podem sobrepor-se, e, desse modo, a situação clínica do paciente não melhora(17).

Segundo se inferiu a partir dos dados desse estudo, Quanto aos aspectos da prevenção e controle da hipertensão arterial, os profissionais estão em condições inadequadas. Portanto, tais resultados denotam a exposição desses profissionais aos riscos ocupacionais. Reforçam, assim, a necessidade de um acompanhamento regular desses profissionais mediante instituição formal dos cuidados a serem implantados, sensibilizando todos os envolvidos no processo do cuidar.

Embora tenham consciência acerca do assunto, os profissionais afirmaram ser imprescindível a existência desse tipo de programa direcionado para as categorias profissionais.

Os componentes da síndrome hipertensiva são muitas vezes fatores de risco cardiovascular independentes. Dessa forma, os esquemas terapêuticos propostos com intenção única de baixar os níveis pressóricos não obtiveram uma redução da morbidade e mortalidade como esperado. Urge, então, desenvolver programas com vistas à prevenção dos fatores de riscos modificáveis e maior 
adesão ao tratamento da hipertensão arterial ${ }^{(17)}$.

Propõem-se a educação em saúde, por ser reconhecida pelo seu potencial para redução de custos junto a diversos contextos da assistência, por favorecer a promoção do autocuidado e o desenvolvimento da responsabilidade sobre decisões relacionadas à saúde, é vista, sobretudo, como Questão importante, entretanto a efetividade de estratégias utilizadas para tal fim vem merecendo debates $^{(18)}$.

A instituição, por meio do SESMT, tem o dever de elaborar e implementar o Programa de Controle Médico de Saúde Ocupacional (PCMSO) para programar as ações preventivas ou corretivas das doenças e riscos ocupacionais. A Norma Regulamentadora (NR) $\mathrm{n}^{07}$ estabelece a obrigatoriedade dessa preparação com o objetivo de promover e preservar a saúde de seus trabalhadores ${ }^{(5)}$.

Algumas destas oportunidades de melhoria contínua podem ser desenvolvidas pelo enfermeiro do trabalho, tais como: programas para gestantes, análise dos indicadores de saúde, atividades educativas baseadas no diagnóstico das doenças ocupacionais do ano anterior, acompanhamento de portadores de necessidades especiais, subnormais e portadores de doenças crônico-degenerativas, programa e registro de imunização no prontuário clínico e individual, reciclagem dos profissionais do SESMT a cada dois anos, incentivo à pesQuisa e divulgação em saúde ${ }^{(19)}$.

Para alcançar os objetivos da saúde ocupacional, é preciso haver uma sistemática de monitoramento dos programas da medicina preventiva. Apesar de a legislação brasileira ser uma das mais avançadas no mundo em relação à proteção do trabalhador contra doenças e acidentes do trabalho, e embora este fato denote grande evolução, nem sempre a legislação é cumprida e fiscalizada( ${ }^{(20)}$.

A enfermagem é uma profissão comprometida com a saúde do ser humano e a Qualidade de vida da pessoa, família e coletividade. Tal como outros profissionais de saúde, o enfermeiro também é vulnerável aos fatores Que predispõem às doenças crônicas não transmissíveis, como a hipertensão arterial.

Em virtude da potencialização dos fatores a Que os profissionais da saúde estão expostos, concentra maior possibilidade de falha no autocuidado desses profissionais, sobretudo Quando relacionado à hipertensão, considerada uma enfermidade Que se instala de maneira silenciosa.

Embora a enfermagem seja reconhecida como uma atividade penosa, por lidar com situações de sofrimento e pela continuidade e disponibilidade exigidas por esse trabalho, a equipe de enfermagem é um agente privilegiado na disseminação de informações e conhecimento sobre a hipertensão arterial e suas complicações, por atuar como intermediário entre o contato de uma rede de atendimento multidisciplinar ${ }^{(6)}$.

Além disso, a equipe de enfermagem executa atividades profissionais Que requerem não apenas a segurança da pessoa, família e comunidade Que estão aos seus cuidados, mas também exigem condições de trabalho Que promovam a sua própria segurança, especialmente a sua Qualidade de vida no trabalho. Para tanto, os profissionais devem desempenhar seu papel com competência técnica, científica, ética e legal.

\section{CONCLUSÕES}

O sucesso para o controle da HAS nos profissionais de enfermagem está ligado ao desenvolvimento de estratégias de educação em saúde e programas preventivos de acompanhamento para esses profissionais. Além disso, ressaltam-se os riscos inerentes ao ambiente de trabalho, no Qual é composto não só da infra-estrutura física e técnica, mas, pelas pessoas Que o utilizam, pois são as pessoas Que contribuem ou não para um ambiente saudável e desejado. As suas atitudes são vitais para a mudança de um ambiente organizacional.

De acordo com o estudo, os profissionais de enfermagem Quanto aos aspectos da prevenção e controle da hipertensão arterial estão em condições inadequadas. Os resultados ora obtidos denotam a exposição desses profissionais aos riscos ambientais e ocupacionais Que possam contribuir a desenvolver tal patologia. Reforça-se, portanto, a necessidade de um acompanhamento regular mediante instituição formal dos cuidados a serem implantados, sensibilizando todos os envolvidos no processo do cuidado.

Quanto ao perfil sociodemográfico da equipe de enfermagem portadora de HAS, constatou-se predomínio das seguintes características: sexo feminino (79,2\%), idade entre 40 a 60 anos $(68,4 \%)$, casado $(41,5 \%)$, cor não-branca $(65,4 \%)$.

Percebe-se Que, como a maioria dos participantes do estudo são mulheres, ressalta-se a importante inclusão de políticas públicas envolvidas na saúde da mulher, além da hipertensão arterial, dando ênfase ao modelo holístico biopsicossocial do cuidar.

Os fatores de riscos modificáveis estavam presentes nos profissionais de enfermagem na seguinte ordem de prevalências: dieta inadequada $(68,5 \%)$, sedentarismo $(62,3 \%)$, etilismo $(43,8 \%)$, tabagismo $(35,4 \%)$, diabetes mellitus $(24,6 \%)$ e dislipidemia (24,6\%). E a hereditariedade, como não-modificável, estava presente em $71,5 \%$ dos profissionais.

Desse modo, além da preocupação de controlar a pressão arterial deve-se enfatizar a prevenção, por ser a melhor terapêutica, sobretudo o combate aos fatores de risco, o Qual deve ocorrer por meio de estratégias educativas de saúde.

Ademais, a instituição possui uma parcela de contribuição relevante no processo de adoecimento do profissional, decorrente da falta do controle de um ambiente corporativo, por necessidade de adeQuação dos seguintes aspectos: definição das políticas de recursos humanos, definições das tarefas a serem realizadas, ofertas de condições físicas adequadas e estímulo frequente à integração das pessoas e equipes.

As políticas públicas envolvendo a saúde do trabalhador contemplam ações de promoção, proteção, e recuperação da saúde. Diante desta realidade, os profissionais com ascendentes índices de fatores de risco devem receber acompanhamento periódico e exercer uma prática de autocuidado favorável à redução das complicações. Essa prática de autocuidado deve ser intensificada, sobretudo, em pessoas já portadoras de HAS.

Em relação à participação em programa de educação em saúde para hipertensão ou doenças cardiovasculares, verificou-se Que $97,7 \%$ dos profissionais entrevistados não participavam, embora concordassem que para minimizar as consequências da hipertensão fosse necessário trabalhar os fatores de risco, prevenindo assim as possíveis complicações.

Então, é necessária a elaboração e promoção de estratégias de incentivo e adoção de medidas para controlar os fatores de risco da hipertensão por parte da instituição e gestores, na expectativa de reduzir as co-morbidades e as mortalidades. É preciso melhorar, particularmente, a Qualidade de vida dos profissionais, considerando 
também a necessidade da implantação de programas de Qualidade de vida, direcionada não só para os clientes, mas para os profissionais, fomentado por uma equipe de saúde ocupacional interdisciplinar, com vistas a evitar as doenças ocupacionais, com práticas voltadas para sensibilização e responsabilidade referente ao autocuidado e estilo de vida. Para isso, é indispensável reconhecer a educação em saúde como um processo contínuo.

Sugere-se, ainda, Que sejam selecionados profissionais de saúde para compor uma equipe de prestação de serviços especializados e seguros aos trabalhadores. Caberá a esta equipe estabelecer uma meta com assistência global Que atenda às necessidades dos servidores e valorize a diversidade de papéis, com vistas à integralidade. Sobretudo, ressalta-se a importância para estimular os gestores a inserir uma política de saúde direcionada para a população feminina e masculina. Espera-se, assim, garantir a adesão de todos os profissionais ao tratamento, sobretudo mediante a sua participação no processo do cuidar, fator essencial.

Recomenda-se, portanto, a realização de outros estudos nesta linha de investigação, sempre com foco no bem-estar do trabalhador de saúde, visando uma abrangência de possibilidades para prestação de cuidados e vigília em relação aos riscos a Que estão expostos esses trabalhadores.

\section{REFERÊNCIAS}

1. Smeltzer SC, Bare BG. Histórico da função cardiovascular. In: Smeltzer SC, Bare BG. Brunner \& Suddarth: tratado de enfermagem médico-cirúrgico. $10^{\text {a }}$ ed. Rio de Janeiro: Guanabara Koogan; 2006. p. 682-700.

2. Sociedade Brasileira Hipertensão. V Diretrizes Brasileiras de Hipertensão Arterial. São Paulo: SBC; 2006.

3. Murofuse NT, Abranches SS, Napoleão AA. Reflexões sobre estresse e burnout e a relação com a enfermagem. Rev Latinoam Enfermagem 2005; 13(2): 255-6I.

4. Ministério da Saúde (BR). Conselho Nacional de Saúde. Resolução 196/96. Decreto n. 93.933 de janeiro de 1987. Estabelece critérios sobre pesquisa envolvendo seres humanos. Bioética 1996; 4(2): 15-25.

5. Ministério da Saúde (BR). Segurança e Medicina do Trabalho. $2^{a}$ ed. São Paulo: Saraiva; 2008.

6. Aquino EMLL, Magalhães LBNC, Araújo MJ, Almeida MdaCCde, Leto JP. Hipertensão arterial em trabalhadoras de enfermagem-padrão de ocorrência, diagnóstico e tratamento. Are Bras Cardiol 200 I; 76: 197-202.

7. Hospital de Messejana Dr. Carlos Alberto Studart Gomes [internet]. Fortaleza; 2009. [citado em 2009 maio 17]. Disponível em: http://www.hm.ce.gov.br.

8. Santos ZMSA, Silva R. Prática do autocuidado vivenciada pela mulher hipertensa: um análise no âmbito da educação em saúde. Rev Bras Enferm 2006; 59(2): 206-11.

9. Beck CLC, Gonzáles RMB, Stekel LMC, Donaduzzi JC. O trabalho da enfermagem em unidades críticas e sua repercussão sobre a saúde dos trabalhadores. Esc Anna Nery Rev Enferm 2006; 10(2): 221-7.

10. Fischer FM, Borges FNS, Rotenberg L, Latorre MRDO, Soares NS, Rosa PLFS, et al. Work ability of health care shift workers: what matters? Chronobiol Int 2006; 23(6): 1 165-79.

I I. Rotenberg L, Portela LF, Marcondes WB, Moreno C, Nascimento CP. Gênero e trabalho noturno: sono, cotidiano e vivência. Cad Saúde Pública 2001; 17(03): 102-13.

12. Fernandes CE, Pinho-Neto JSL, Gebara OCE, Santos Filho RD, Pinto Neto AM, Pereira Filho AS, et al. I Diretriz Brasileira sobre Prevenção de Doenças Cardiovasculares em Mulheres Climatéricas e a Influência da Terapia de Reposição Hormonal da Sociedade Brasileira de Cardiologia e da Associação Brasileira do Climatério. Are Bras Cardiol 2008; 91(I supl 1): I-23.

13. Victora CG, Piccini RX. Prevalência da Hipertensão Arterial Sistêmica em Área Urbana no Sul do Brasil: prevalência e fatores de risco. Rev Saúde Pública 2004; 28(4): 261-7.

14. Silva JLL, Souza SL de. Fatores de risco para hipertensão arterial sistêmica versus estilo de vida docente. Rev Eletrônica Enfermagem 2004; 6(3):330-5.

15. Sousa IA, França ISX. Prevalência de Hipertensão Arterial em pessoas com mobilidade física prejudicada: implicações para a enfermagem. Rev Bras Enferm 2008; 6I (6): 8I6-2I.

16. Kasinsinski N, Stefanini E, Carvalho AC. Guias de Medicina Ambulatorial e Hospitalar de Cardiologia. São Paulo: Manole; 2004.

17. Mano R. Hipertensão Arterial Sistêmica. Manuais de Cardiologia. Rio de Janeiro; 2009. [citado em 2010 Nov 13]. Disponível em: http://www.manuaisdecardiologia.med.br/has/has.htm

18. Chaves ES, Lúcio IML, Araújo TL, Damasceno MMC. Eficácia de programas de educação para adultos portadores de Hipertensão Arterial. Rev Bras Enferm 2006; 59(4): 543-7.

19. Lima MMR, Dantas RA, Pagliuca LMF, Almeida PC. Programa de Controle Médico de Saúde Ocupacional em Companhias Elétricas Brasileiras. Rev RENE 2007; 8(3):61-68.

20. Bulhões II. Enfermagem do Trabalho. Rio de Janeiro: Ideas; 1986. 